\title{
Experimental Demonstration of Centralized and Distributed Impairment-Aware Control Plane Schemes for Dynamic Transparent Optical Networks
}

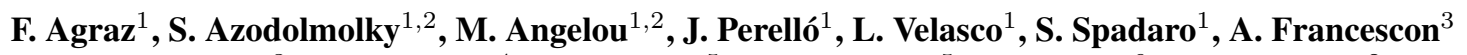 \\ C.V. Saradhi ${ }^{3}$, Y. Pointurier ${ }^{4}$, P. Kokkinos ${ }^{5}$, E. Varvarigos $^{5}$, M. Gunkel ${ }^{6}$ and I. Tomkos ${ }^{2}$ \\ (1) Universitat Politècnica de Catalunya (UPC), Barcelona, Spain; (2) Athens Information Technology (AIT), Athens, Greece; \\ (3) Create-Net, Trento, Italy; (4) Alcatel-Lucent, Bell Labs, Nozay, France; (5) Research Academic Computer Technology \\ Institute (RACTI), Patras, Greece; (6) Deutsche Telekom AG, Darmstadt, Germany; e-mail address: itom@ait.edu.gr
}

\begin{abstract}
We demonstrate and compare distributed and centralized impairment-aware control plane schemes for transparent optical networks with dynamic traffic. Experimental results show that distributed scheme yields one fifth of setup time required by previously reported alternatives.

(c) 2010 Optical Society of America

OCIS codes: (060.4250) Networks; (060.4510) Optical communications
\end{abstract}

\section{Introduction}

In transparent optical networks, the signal experiences the impact of various phenomena that degrade its quality. Considering the impact of physical layer impairments (PLIs) on transparent [1] and highly dynamic optical networks [2] has received much attention recently. The main innovation of the DICONET project [3] is the development of a dynamic Network Planning and Operation Tool (NPOT) that incorporates real-time assessments of the optical layer performance into Impairment Aware Routing and Wavelength Assignment (IA-RWA) algorithms. The NPOT is integrated into a unified extended Generalized Multi-Protocol Label Switching (GMPLS)-based control plane. The work in [1] reported the result of a centralized integration scheme for transparent networks considering various PLIs, while [4] only investigated a distributed GMPLS integration for translucent networks.

This paper demonstrates the performance of centralized and distributed impairment-aware control plane approaches over a realistic 14-node experimental test-bed under dynamic traffic conditions.Initial simulation results were reported in [5] and experimental results are reported here.The test-bed integrates the developed NPOT engine, the extended GMPLS control protocols required for supporting the innovative DICONET solutions and the various communication protocols to allow all DICONET building blocks run in an orchestrated fashion. To the best of our knowledge, for the first time, impairment-aware control plane schemes with integrated real-time Quality of Transmission (QoT) estimator are demonstrated.

\section{Impairment-aware lightpath provisioning: DICONET approaches}

In the centralized approach (Fig. 1, left), the NPOT carries out the impairment-aware lightpath computation and failure handling functionalities, while the Optical Connection Controllers (OCCs) execute the extended GMPLS protocol stack and interface to the actual optical nodes in the test-bed. To this goal, a TCP-based messaging protocol has been developed to facilitate the communication between OCCs and the centralized NPOT. Upon the arrival of a connection request, the source OCC contacts the centralized NPOT to request a path computation. Next the request is forwarded to the online IA-RWA module [6], which is responsible for performing the path computation for the given request. This module utilizes the QoT estimator (Q-Tool) and the information of the global Physical Parameters and Traffic Engineering Databases (gPPD and gTED), which describe the network topology and the physical layer characteristics completely. In particular, Q-Tool is the module within NPOT that quantifies the impact of the PLIs on the lightpaths' QoT. Using a method that combines numerical simulations and analytical approximations, the Q-Tool considers the dominant linear and non-linear PLIs [7], whose effect is reflected in the estimated Q-factor. The same QoT estimator is used in the distributed scheme.

When the NPOT finds a lightpath with guaranteed QoT (Q-factor value above a pre-defined threshold), the lightpath is returned back to the source OCC and it is established from source to destination using standard RSVP-TE signaling protocol. Upon successful establishment of a lightpath, the global PPD and TED in the NPOT and the local PPDs and TEDs in every OCC in the network are updated using the extended OSPF-TE protocol. Finally, the source OCC updates the NMS. In case of lack of resources or unacceptable QoT, the demand is blocked and the source OCC informs the Network Managent System (NMS) accordingly. When a link failure occurs, the downstream optical nodes 


\section{PDPD5.pdf}
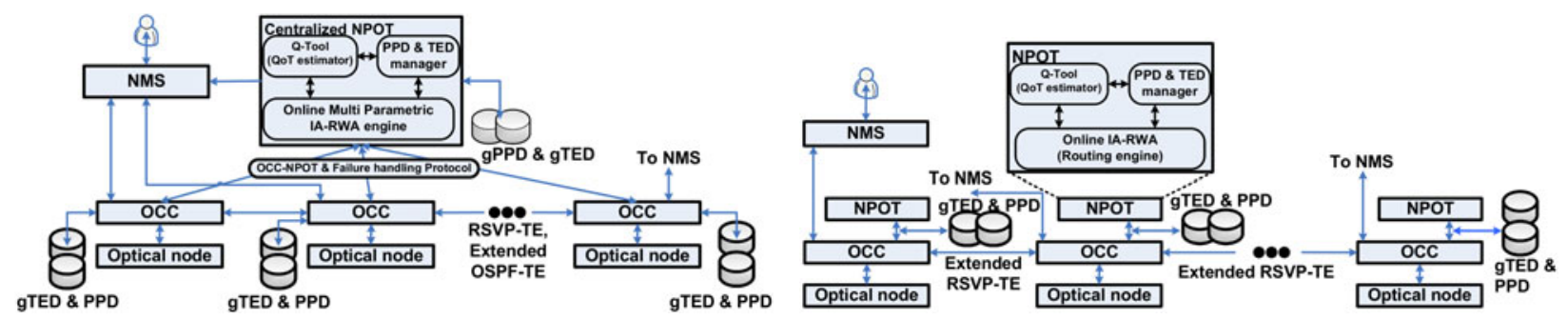

Fig. 1. DICONET Project Impairment-Aware control plane approaches: centralized (left) and distributed (right)

detect it and send alarms to their OCCs, which forward the failure notification to the centralized NPOT. In order to restore the affected lightpaths and to avoid using the failed network resources, the centralized NPOT localizes the failure, updates both gTED and gPPD, and computes the backup paths. Then, the source OCCs trigger the signaling protocol for the actual lightpath establishment.

In the distributed approach (Fig. 1, right), both RSVP-TE and OSPF-TE protocols have been extended to consider PLIs, providing a compromise between network performance, control overhead and complexity. The OSPF-TE protocol has been extended to enable the dissemination of wavelength availability information. The RSVP-TE signaling protocol has been extended to collect, through the PATH message, real-time information of the PLIs during the connection establishment process. Here, each node in the network runs an instance of the distributed NPOT, which is connected to the OCC via the NPOT-OCC communication protocol. Upon receiving a new connection request, the source OCC requests the online IA-RWA module of the NPOT to compute $k$-routes from source to destination. The wavelength availability information stored in the gTED is used for route computation. Once these $k$-routes are computed, the source OCC triggers the extended RSVP-TE protocol to initiate the lightpath establishment using the first candidate route. The RSVP-TE PATH message collects the PLIs from source to destination along the candidate path, and the destination node requests for a QoT estimation from its corresponding NPOT. If the QoT of the candidate lightpath is acceptable, then the destination nodes of the potentially disrupted lightpaths (i.e., those lightpaths sharing at least one optical section with the candidate one) are notified to request for a QoT estimation from their respective NPOTs. This verification step makes sure that the Q-factor of the already established lightpaths remains above the required threshold in spite of the establishment of the new lightpath. If there is no violation, the destination nodes of these active lightpaths update their local databases with the new lightpath information and respond back to the destination node of the candidate one. If the re-computed Q-factor values are above the threshold, an RSVP-TE RESV message is sent back to the source node and the actual cross-connections are properly configured. Otherwise, an RSVP-TE PATH_ERR is returned back, and the source node tries another candidate lighpath. If none of the $k$-candidates meet the required QoT the request is finally blocked.

\section{Experimental results and discussion}

The performance of the proposed approaches has been validated on a 14-node network test-bed located at the UPC premises in Barcelona. The test-bed describes the same topology as the Deutsche Telekom (DT) network (Fig. 2, left), where 10 bidirectional wavelengths per link have been assumed. Each network node is composed of an OCC and a WSS-based OXC emulator, both interconnected through the Connection Controller Interface (CCI). In particular, OCCs implement the extended versions of RSVP-TE and OSPF-TE for carrying PLI information, as detailed in section 2. Regarding the traffic characteristics, uniformly distributed lightpath requests arrive to the network following a Poisson process. Moreover, lightpath holding times (HTs) are exponentially distributed with mean $600 \mathrm{~s}$. Different loads are thus generated by modifying the connection inter-arrival times (IATs) accordingly (load $=$ HT/IAT).

Fig. 2 (center) depicts the setup delay experienced by the incoming requests, depending on whether the distributed or centralized approaches are deployed in the network. As seen, the distributed approach yields lower setup times in all experiments and the difference increases with the offered load to the network. To explain this, note that in the centralized scheme only one route computation is allowed at the same time. Furthermore, a sufficient amount of time must be left between two consecutive route computations in order to let the centralized NPOT be fed with the new wavelength availability and PLI information. Otherwise, subsequent routes might be computed with inaccurate link state information. Therefore, the centralized NPOT scheduler must delay new incoming requests until the signaling and the respective flooding of the previous connection establishment has been completed (around $2 \mathrm{~s}$ in the test-bed). In contrast, the distributed approach can benefit from parallel lightpath establishments, as the Q-factor values of the new LSP and the involved active ones are computed during the signaling process. This eventually results into very 


\section{PDPD5.pdf}
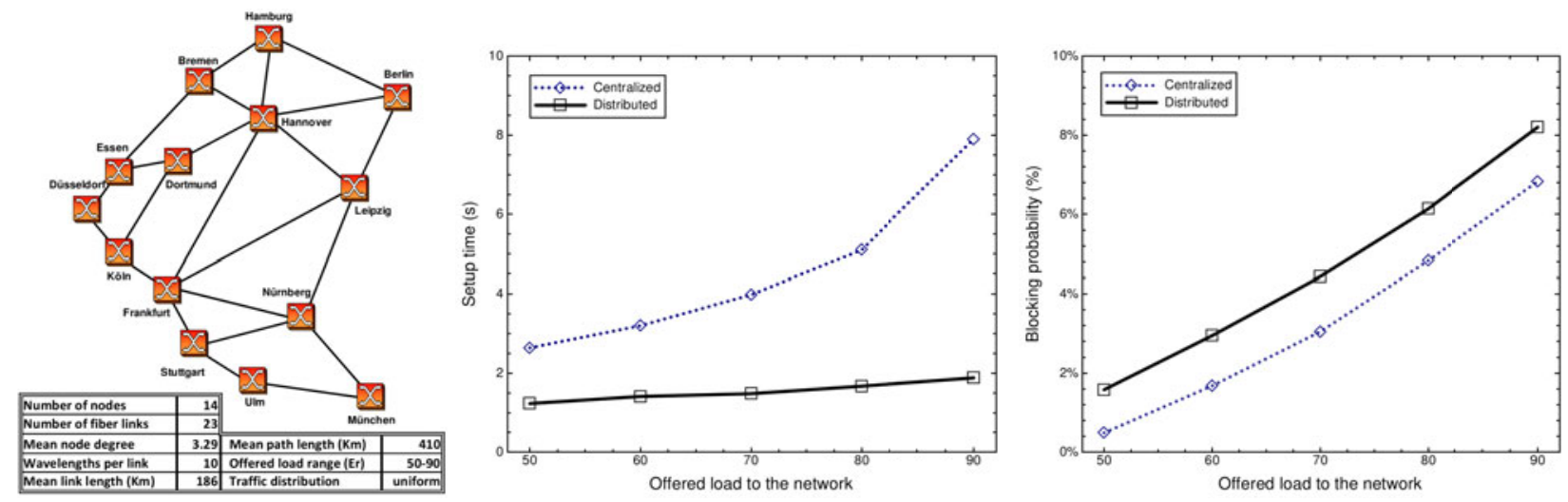

Fig. 2. Experimental scenario and results: Deutsche Telekom (DT) Network (left); lightpath setup time vs. offered load (center); blocking probability vs. offered load (right). All results in the figures have been obtained by averaging 10.000 incoming requests.

attractive connection setup times, around $1.8 \mathrm{~s}, 1 / 5$ th of the setup time reported in [1].

In line with the lightpath setup delay, we have conducted additional experiments to assess the performance of NPOT when handling failure restoration scenarios. These results focus on the centralized approach as, in this case, the NPOT is enhanced with a failure localization module that collects the alarms from all OCCs detecting Loss-of-Light (LoL) traps. Note that in the distributed approach, the alarms upon LoL trap detection are only sent to the respective NPOT, thus requiring the network an additional protocol for failure isolation (e.g., GMPLS LMP protocol). For the measurements, we cut the fiber connecting Hamburg and Stuttgart, which supports one active lightpath. The experimentally measured time required to restore the failed lightpath is around $1.3 \mathrm{~s}$.

Finally, Fig. 2 (right) illustrates the network blocking probability (BP) resulting from distributed and centralized approaches. Particularly, $k=2$ shortest routes are allowed in the distributed case. As shown, a centralized approach leads to lower BP than the distributed solution. In fact, end-to-end routes in the latter are computed only with wavelength availability information. These routes lead in some occasions to unacceptable Q-factor of candidate or potentially disrupted lightpaths and hence need to be blocked. In contrast, route computation in the centralized approach relies on complete and updated wavelength availability and PLI information, making the computed routes satisfy the requested Q-factor values.

\section{Conclusions}

This paper presented centralized and distributed control plane approaches for impairment-aware transparent optical networks. From the experimental evaluation, the distributed approach provides one fifth of the lightpath setup time than that of previously reported (centralized) alternatives, also outperforming our centralized approach especially for high traffic loads. For low traffic loads, however, our centralized approach results in reduced lightpath blocking ratio and similar setup time delays than the distributed solution, thus being more appropriate in such scenarios. Efforts in DICONET will be devoted to further reduce lightpath setup time to milliseconds' time-scales by means of FPGA hardware acceleration.

The authors thank the other DICONET partners (ADVA, ECI, Huawei, IBBT, TPT, and Univ. of ESSEX) for their valuable inputs. This work has been supported by the European Commission through the FP-7 DICONET Project.

\section{References}

[1] T. Tsuritani et al., "Optical Path Computation Element Interworking with Network Management System for Transparent Mesh Networks," in Proc. of OSA OFC/NFOEC 2008, NWF5, 2008.

[2] A. Chiu, et al., "Network Design and Architectures for Highly Dynamic Next-Generation IP-over-Optical Long Distance Networks," in Proc. of OSA OFC/NFOEC 2009, NThF3, 2009.

[3] S. Azodolmolky, et al., A Dynamic Impairment-Aware Networking Solution for Transparent Mesh Optical Networks," IEEE Comm. Mag., vol. 47, no. 5, May 2009.

[4] R. Martinez et al., "Experimental GMPLS Routing for Dynamic Provisioning in Translucent Wavelength Switched Optical Networks," in Proc. of OSA OFC/NFOEC 2009, NTuB4, 2009.

[5] I. Tomkos, et al., "Impairment Aware Networking and Relevant Resiliency Issues in All-Optical Networks", Proc. of ECOC 2008.

[6] P. Kokkinos, et al., "Multi-Parametric Online RWA Based on Impairment Generating Sources," GLOBECOM 2009. IEEE , vol., no., pp.1-7, Nov. 30 2009-Dec. 42009.

[7] P. Pavon-Marino, et al., "Offline Impairment Aware RWA Algorithms for Cross-Layer Planning of Optical Networks," IEEE/OSA Journal Lightw. Technol., 27 (12), pp. 1763-1775, 2009. 